\title{
Stochastic Bifurcations induced by correlated Noise in a Birhythmic van der Pol System
}

\author{
R. Mbakob Yonkeu, ${ }^{1}$ R. Yamapi ${ }^{2}$ G. Filatrella, ${ }^{3}$ and C. Tchawoua ${ }^{1}$ \\ ${ }^{1}$ Laboratory of Mechanics and Materials, Department of Physics, \\ Faculty of Science, University of Yaoundé I, Box 812, Yaoundé, Cameroon. \\ ${ }^{2}$ Fundamental Physics Laboratory, Physics of Complex System group, Department of Physics, \\ Faculty of Science, University of Douala, Box 24157 Douala, Cameroon. \\ ${ }^{3}$ Department of Sciences and Technologies and Salerno unit of CNISM, \\ University of Sannio, Via Port'Arsa 11, I-82100 Benevento, Italy.
}

(Dated: 5 August 2015)

\begin{abstract}
We investigate the effects of exponentially correlated noise on birhythmic van der Pol type oscillators. The analytical results are obtained applying the quasi-harmonic assumption to the Langevin equation to derive an approximated Fokker-Planck equation. This approach allows to analytically derive the probability distributions as well as the activation energies associated to switching between coexisting attractors. The stationary probability density function of the van der Pol oscillator reveals the influence of the correlation time on the dynamics. Stochastic bifurcations are discussed through a qualitative change of the stationary probability distribution, which indicates that noise intensity and correlation time can be treated as bifurcation parameters. Comparing the analytical and numerical results, we find good agreement both when the frequencies of the attractors are about equal or when they are markedly different.

Keywords::Stochastic bifurcation; colored noise; birhythmic system; Fokker-Planck equation

Corresponding author: E-mail address: filatrella@ unisannio.it (G. Filatrella).

To appear in: Commun. Nonlinear Sci. Numer. Simulat.

PACS numbers:

05.10.Gg Stochastic analysis methods (Fokker-Planck, Langevin, etc.

82.40.Bj Oscillations, chaos, and bifurcations

05.45.-a Nonlinear dynamics and chaos

82.40.-g Chemical kinetics and reactions: special regimes and techniques
\end{abstract}

\section{INTRODUCTION}

When studying a phenomenon, one is generally interested in overriding effects. Nonlinear science has been used to account for some phenomena in physics, biology and modeling of social facts that could not be otherwise explained, even at a qualitative level. Among these phenomena, we can number self-sustained oscillations and noise activated transitions. A paradigmatic model for oscillating systems is the van der Pol circuit, named after the work of Balthazar van der Pol, who introduced relaxation oscillations to describe the cycles produced by self-sustained oscillating systems such as the triode circuit.[1-3]. With its many variants, van der Pol oscillators have served as a paradigm in various physical, chemical, and biological processes [4-6]. A special merit of van der Pol has been to propose a dimensionless (or reduced) equation in the terminology of Curie [1, 7], for it can be used to explain various system regardless their origins. The van der Pol system was not the first one where self oscillating behaviors were observed, but this is the first reduced equation in Curie's sense. With its graphical representation [8] van der Pol also contributed to change the way in which these nonlinear phenomena had to be investigated.

The oscillatory motion can be influenced by internal or external noise [9-11] and the resulting interplay between nonlinearities and noise can induce transitions similar to the standard transition between two stable points [12, 13]. In the case of self-sustained oscillations, one observes a transition between two stable orbits rather than two points. However, it has been shown that the escape is indeed of the Kramers' type for uncorrelated noise [14, 15] even in presence of time delay [1618], and it can be ascribed to the existence of a quasipotential or pseudopotential [19, 20]. The quasipotential allows to estimate the energy barriers, and indicates that for most parameters, a single attractor is dominantly stable [14, 15], while the other is characterized by a small energy barrier. The effect of a finite correlation time has also been investigated, and it has been found that, as expected for static potential, also the periodic orbits are stabilized by the finite correlation [21].

Noise, however, can produce also effects that are more radi$\mathrm{cal}$, for it can induce a structural (or topological) change of the solutions [22]. This is the case when noise induces a stochastic bifurcation, that may be characterized with a qualitative change of the stationary probability distribution. Moreover, noise can stabilize unstable equilibria and shift bifurcations, or it can even induce new stable states that have no deterministic counterpart (for instance noise can excite internal modes of oscillation and it can even enhance the response of a nonlinear system to external signals [23-25]). When the noise driven stochastic differential equation is colored, the nature of the stochastic process becomes non-Markovian and cannot be treated with the standard Fokker-Planck techniques. The study of stochastic nonlinear systems driven by colored noise has been undertaken by a number of investigation [26-28]. On a general ground correlated noise appears as the result of the gross approximation of a system over some finite scale that masks the effect of slow variables [27], as it has been argued in ecology [29] or electronic circuits [24]. Birhythmic oscillators 
are no exception, as they emerge in biochemical reactions [30] or sleeping patterns [4, 6] where such coarsening could occur. In many treatments, one is essentially concerned with an equilibrium thermal bath at a finite temperature, which stimulates the reaction coordinate to cross the activation energy barrier. In this paper, we wish to investigate the transition between orbits [31-34], characterized by different frequencies (and hence birhythmic systems). To do so we analytically treat the effects of Gaussian correlated noise on a special van der Pol oscillator system that displays a birhythmic behavior, for the two coexisting attractors characterized by different frequencies. We search the relation between stochastic bifurcations and noise correlation time on the dynamical properties, taking the system parameters and the statistical characteristics of the noise (e.g., noise intensity and noise correlation time) as bifurcation parameters. Our approach of the bifurcations is based on the stochastic averaging method [5], that is separating fast and slow variables of the oscillator.

The work is organized as follows. In Sect. II we discuss the model and the numerical approach to the discretized equations. In Sect. III we derive an analytic approximation that includes the effects of a finite correlation time. Section IV compares the analytical and numerical results. Section $\square$ concludes.

\section{THE CORRELATED NOISY MODEL AND BIRHYTHMIC PROPERTIES}

In this Section we present the van der Pol model that we use as a prototype of birhythmic model. We do so first presenting the electrical equivalent circuit and then deriving the normalized equations.

\section{A. The correlated noisy model}

The model used in our analysis can be reproduced with electronic circuits. An example of equivalent circuit is shown in Fig. 1 It consists of electronic multipliers $M_{i}(i=$ $1, \ldots, 4)$, integrators that are operational amplifiers with a feedback capacitor, and sommators realized by operational amplifiers with multiple input resistors. Using Millman law, the characteristic of each component and the contributions of the electrical voltage $V_{e}$ supplied by an external source (that in this study is assumed to be a random term) we find for the potential at the left of the point $S$ :

$$
V_{s}=E \frac{R_{13}}{R_{12}}-\frac{R_{13}}{R_{10}} \frac{V^{2}}{K}+\frac{R_{13}}{R_{9}} \frac{V^{4}}{K^{3}}-\frac{R_{13}}{R_{11}} \frac{V^{6}}{K^{5}} .
$$

where $K$ is a scaling factor, which has the dimension of voltage. The multiplicator $M_{1}$ gives the end voltage (the dots denote the time derivative):

$$
\begin{aligned}
& \int\left[\frac{V_{e}}{R_{1} C_{1}}-\frac{V}{R_{3} C_{1}}+\left(\frac{R_{13} E}{R_{12}}-\frac{R_{13}}{R_{10}} \frac{V^{2}}{K}+\frac{R_{13}}{R_{9}} \frac{V^{4}}{K^{3}}-\frac{R_{13}}{R_{11}} \frac{V^{6}}{K^{5}}\right) \frac{\dot{V}}{K}\right] \frac{d t^{\prime}}{R_{2} C_{1}} \\
= & \dot{V} .
\end{aligned}
$$

If we take the time derivative of Eq.(2), we get

$$
R_{2} C_{1} \ddot{V}-\frac{E}{K} \frac{R_{13}}{R_{12}}\left(1-\frac{R_{12}}{R_{10} E} \frac{V^{2}}{K}+\frac{R_{12}}{R_{9} E} \frac{V^{4}}{K^{3}}-\frac{R_{12}}{R_{11} E} \frac{V^{6}}{K^{5}}\right) \dot{V}+\frac{1}{R_{3} C_{1}} V=\frac{1}{R_{1} C_{1}} V_{e} .
$$

It is convenient to introduce the variables $t^{\prime}=\omega_{0} t$ and $V=V_{0} x$, where $V_{0}$ is the reference voltage. With the constraint $R_{12} V_{0}^{2}=R_{10} E K$, Eq. (3) becomes the following non-dimensional differential equation:

$$
\ddot{x}-\mu\left(1-x^{2}+\alpha x^{4}-\beta x^{6}\right) \dot{x}+x=\eta(t),
$$

where

$$
\begin{gathered}
\mu=\frac{R_{13} E}{K R_{12} R_{2} C_{1} \omega_{0}} ; \quad \alpha=\frac{R_{12} V_{0}^{4}}{R_{9} E K^{3}} ; \quad \beta=\frac{R_{12} V_{0}^{6}}{R_{11} E K^{5}} ; \\
\omega_{0}^{2}=\frac{1}{R_{2} R_{3} C_{1}^{2}} ; \quad \eta(t)=\frac{R_{3}}{R_{1} V_{0}} V_{e} .
\end{gathered}
$$

A similar equation was originally obtained by van der Pol in the analysis of a triode, when the triode was approximated with a cubic function truncating the Taylor-Mclaurin series expansion [2, 3]. Analogously, one can consider a further expansion to the $5^{\text {th }}$ order [35, 36] to retrieve an equation as the above (3).

Equation (3), even when $\eta(t) \neq 0$, is of the van der Pol type equation, inasmuch most of the practical applications are driven by weak noise intensities. It is also worth mentioning that van der Pol provided a stability criterion for periodic solutions when the coefficients are sufficiently small. This equation is also used to model coherent oscillations in biological system [6, 30, 35]. In the context of enzymatic reactions [30] the parameters $\alpha$ and $\beta$ are positive parameters which measure the degree of tendency of the system to a ferroelectric instability compared to its electric resistance, while $\mu$ is the parameter that tunes non linearity. 


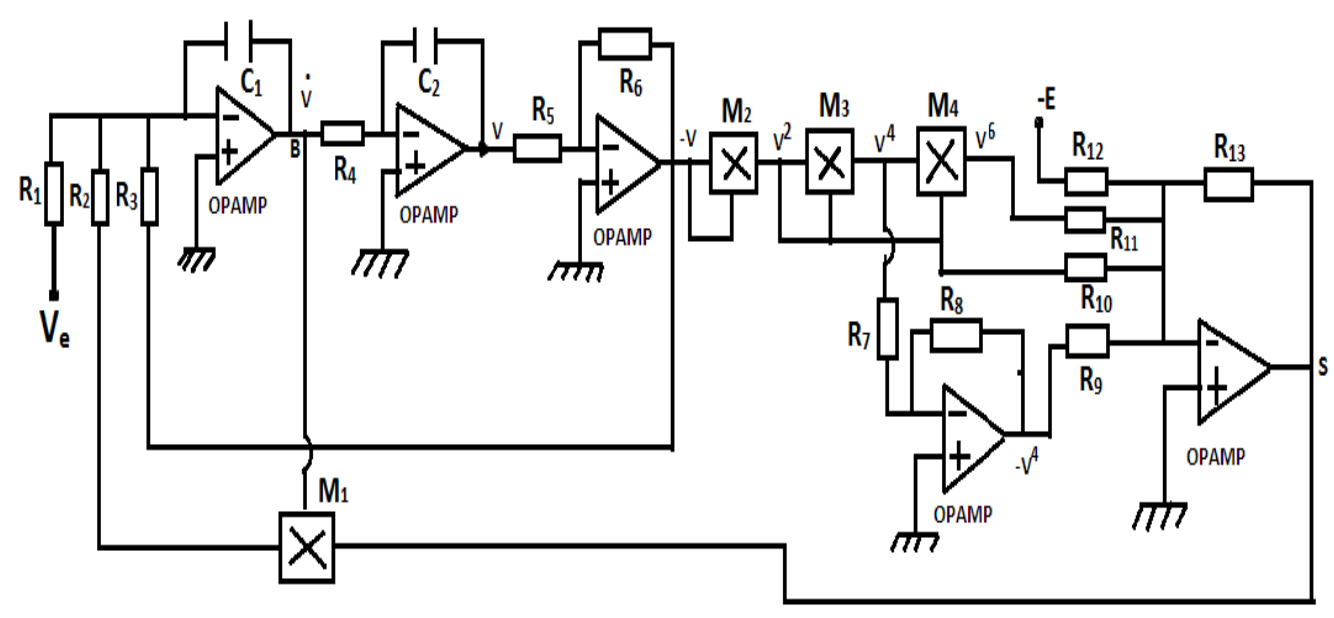

FIG. 1. Circuit diagram for an electronic circuit approximately described by a birhythmic van der Pol type equation. Here the $M_{i}$ 's are analog multipliers circuits while the operation amplifiers with feedback capacitors perform analogue integrations.

We assume that both the noise $\eta(t)$ is stationary and independent of the memory Kernel, thus one cannot apply the fluctuation-dissipation theorem. We also assume that the random excitation $\eta(t)$ is a zero average:

$$
\langle\eta(t)\rangle=0
$$

correlated noise, so we have

$$
\langle\eta(t), \eta(\tilde{t})\rangle=D \lambda \exp [-\lambda(t-\tilde{t})] .
$$

(the parameter $D$ is the intensity of the noise and $\tau=\lambda^{-1}$ is the correlation time for the colored noise.)

The correlated noise $\eta(t)$ can be generated as the solution of the Langevin equation [37]:

$$
\dot{\eta}(t)=-\lambda \eta(t)+\lambda g_{w}(t)
$$

where $g_{w}(t)$ is a standard Gaussian distribution of unit variance.

To complete the description, one further specifies the distribution of initial $\eta_{0}$ values in Eq.(7a), denoted by the parenthesis $\{\ldots\}$ :, which is essential for the stationary correlation ( one should consider different initial conditions, with different initializations of the correlated noise, a caution not necessary for uncorrelated noise):

$$
\{\langle\eta(t) \eta(\tilde{t})\rangle\}=D \lambda \exp (-\lambda|t-\tilde{t}|) .
$$

The distribution of the initial values is given by:

$$
P\left(\eta_{0}\right)=\frac{1}{(2 \pi D \lambda)} \exp \left[-\frac{\eta_{0}^{2}}{2 D \lambda}\right] .
$$

The overall noise intensity $D$ is the zero-frequency part of the power spectrum of the (stationary) noise source $\eta(t)$

$$
2 D=\int_{-\infty}^{\infty}|\langle\eta(t) \eta(0)\rangle| d t
$$

The parameter $\tau$ can be also referred to the intrinsic correlation time:

$$
\tau=\frac{2 D}{\left\langle\eta^{2}\right\rangle}
$$

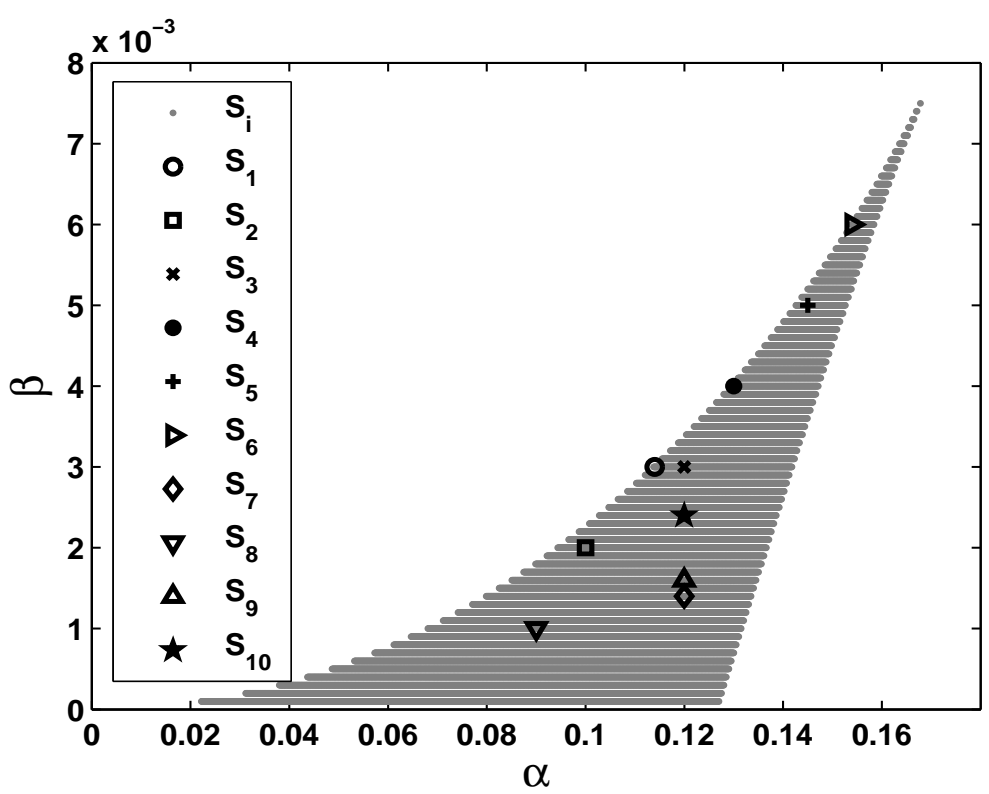

FIG. 2. Parameters domain for the existence of a single limit cycle (white area) and three limit cycles (colored area) with $\mu=0.01$ and the parameter $S_{i}(\alpha, \beta)(i=1,2,3,4,5,6)$ where $\Omega_{1}=\Omega_{3}=1$ (Table I) and $S_{i}(i=7,8,9,10)$ where $\Omega_{1} \neq \Omega_{3}$ (Table II].

The analysis of the noisy dynamical flow involves a study in terms of a two-parameter space $(D, \tau)$. Accurate approximation schemes for colored noise are only valid in the asymptotic 


\begin{tabular}{|l|c|c|c|}
\hline$S_{i}(\alpha, \beta)$ & Amplitudes $A_{i}$ of the orbits & Frequencies $\Omega_{i}$ of the orbits & Periods $P_{i}$ of the orbits \\
\hline \multirow{5}{*}{$S_{1}(0.114 ; 0.003)$} & $A_{1}=2.3772$ & $\Omega_{1}=1.0021$ & $P_{1}=6.270$ \\
& $A_{2}=5.0264$ & $\Omega_{2}=1.0011$ & $P_{2}=6.275$ \\
& $A_{3}=5.4667$ & $\Omega_{3}=1.0231$ & $P_{3}=6.141$ \\
\hline \multirow{5}{*}{$S_{2}(0.1 ; 0.002)$} & $A_{1}=2.3069$ & $\Omega_{1}=0.9870$ & $P_{1}=6.366$ \\
& $A_{2}=4.8472$ & $\Omega_{2}=1.0001$ & $P_{2}=6.275$ \\
& $A_{3}=7.1541$ & $\Omega_{3}=0.97123$ & $P_{3}=6.468$ \\
\hline \multirow{5}{*}{$S_{3}(0.12 ; 0.003)$} & $A_{1}=2.4269$ & $\Omega_{1}=0.9850$ & $P_{1}=6.379$ \\
& $A_{2}=4.2556$ & $\Omega_{2}=0.9990$ & $P_{2}=6.289$ \\
& $A_{3}=6.3245$ & $\Omega_{3}=0.9865$ & $P_{3}=6.369$ \\
\hline \multirow{5}{*}{$(0.13 ; 0.004)$} & $A_{1}=2.4903$ & $\Omega_{1}=1.0002$ & $P_{1}=6.282$ \\
& $A_{2}=4.4721$ & $\Omega_{2}=1.0001$ & $P_{2}=6.282$ \\
& $A_{3}=5.0791$ & $\Omega_{3}=0.9991$ & $P_{3}=6.289$ \\
\hline \multirow{3}{*}{$(0.145 ; 0.005)$} & $A_{1}=2.6605$ & $\Omega_{1}=1.0002$ & $P_{1}=6.282$ \\
& $A_{2}=3.8305$ & $\Omega_{2}=1.0001$ & $P_{2}=6.282$ \\
& $A_{3}=4.9643$ & $\Omega_{3}=1.0005$ & $P_{3}=6.280$ \\
\hline \multirow{5}{*}{$S_{6}(0.154 ; 0.006)$} & $A_{1}=2.7864$ & $\Omega_{1}=0.9992$ & $P_{1}=6.288$ \\
& $A_{2}=3.8821$ & $\Omega_{2}=1.0001$ & $P_{2}=6.282$ \\
& $A_{3}=4.2698$ & $\Omega_{3}=1.0002$ & $P_{3}=6.282$ \\
\hline
\end{tabular}

TABLE I. Characteristics of the approximated [35] limit cycles, as per Eq. [11], of the birhythmic van der Pol system, Eq. 21] for the noiseless case $(D=0)$ when the two stable frequencies are about equal (i.e. $\left.\Omega_{1} \simeq \Omega_{3}\right)$. All data refer to the case $\mu=0.01$.

\begin{tabular}{|l|c|c|c|}
\hline$S_{i}(\alpha, \beta)$ & Amplitudes $A_{i}$ of the orbits & Frequencies $\Omega_{i}$ of the orbits & Periods $P_{i}$ of the orbits \\
\hline \multirow{5}{*}{$S_{7}(0.12 ; 0.0014)$} & $A_{1}=2.491378$ & $\Omega_{1}=1.00210$ & $P_{1}=6.2698$ \\
& $A_{2}=3.52558$ & $\Omega_{2}=0.99994$ & $P_{2}=6.2834$ \\
& $A_{3}=10.88605$ & $\Omega_{3}=0.57300$ & $P_{3}=10.9651$ \\
\hline \multirow{3}{*}{$S_{8}(0.09 ; 0.001)$} & $A_{1}=2.26969$ & $\Omega_{1}=0.99986$ & $P_{1}=6.2839$ \\
& $A_{2}=4.59373$ & $\Omega_{2}=0.99974$ & $P_{2}=6.2847$ \\
& $A_{3}=10.85109$ & $\Omega_{3}=0.68107$ & $P_{3}=9.2252$ \\
\hline \multirow{3}{*}{$S_{9}(0.12 ; 0.0016)$} & $A_{1}=2.48185$ & $\Omega_{1}=1.00034$ & $P_{1}=6.2809$ \\
& $A_{2}=3.58637$ & $\Omega_{2}=0.99993$ & $P_{2}=6.2845$ \\
& $A_{3}=10.04878$ & $\Omega_{3}=0.72935$ & $P_{3}=9.2352$ \\
\hline \multirow{3}{*}{$S_{10}(0.12 ; 0.0024)$} & $A_{1}=2.44836$ & $\Omega_{1}=0.98930$ & $P_{1}=6.3510$ \\
& $A_{2}=3.88657$ & $\Omega_{2}=0.99989$ & $P_{2}=6.2837$ \\
& $A_{3}=7.67464$ & $\Omega_{3}=0.86703$ & $P_{3}=7.2466$ \\
\hline
\end{tabular}

TABLE II. Characteristics of the approximated [35] limit cycles, as per Eq. (11], of the birhythmic van der Pol system, Eq. (21) for the noiseless case $(D=0)$ when the two stable frequencies are different, (i.e. $\left.\Omega_{1} \neq \Omega_{3}\right)$. All data refer to the case $\mu=0.01$.

limits of one the both parameters $D$ and/or $\tau$, as discussed in Ref. [27].

\section{B. Birhythmic properties}

The properties of the dynamical attractors of the modified van der Pol model (3) can be analyzed as follows. One first assumes that the periodic solutions of Eq. (3) are represented by [35]

$$
x(t)=A_{i} \cos \Omega_{i} t .
$$

The amplitudes $A_{i}$ and the frequencies $\Omega_{i}$ can be analytically approximated [35]; in this scheme the amplitude $A_{i}$ is inde- pendent of the coefficient $\mu$, that only enters in the frequency $\Omega_{i}$ of the orbits. The number of cycles of the modified van der Pol system Eq. (3) is determined by the parameters $\alpha$ and $\beta$. It is found that in some region of the parameter space one obtains three limit cycles (two of them are stable, and one is unstable). For each of the two stable limit cycles it corresponds to a different frequency, and hence the system is birhythmic. The parameter plane $(\beta-\alpha)$ is represented in Fig.2, where a shadowed area denotes the portion of the region where birhythmicity occurs [35, 36]. At the border of the shadowed area one observes the passage from a single limit cycle to three limit cycles. The limit cycles exhibit very similar frequencies in the region of low $\alpha$ (Table I) and clearly different frequencies when $\alpha$ increases (Table $\amalg$. By way of 
conclusion of this part, we have described the equation of motion of the system of Fig. 1 when the current is truncated to the fifth order. The system is known to be birhythmic, and the main properties, approximating the solution as in Eq.(11) are summarized in Tables $\square$ and $\Pi$.

\section{ANALYTIC ESTIMATES}

In this Section we derive the properties of the attractors described in Sect. Пwhen subject to correlated noise.

\section{A. Residence times of the attractors}

The evolution of the parameter $x(t)$ is subjected to the influence of random forces, which permanently tend to destabilize it. Random fluctuations, in the case of three attractors (two stable and one unstable) can induce a transition between the two stable attractors [14]. The ubiquitous problem of noise induced escape from a metastable state is characterized by a rate; when the phenomenon occurs on a long time scale compared to dynamic time scales of the localized solution it assumes the features of an Arrhenius like behavior for a dynamical attractor [19, 20], and in particular in van der Pol birhythmic systems [14, 15].

Fig. 3 is an illustration of the escape process from a state of local stability via noise-assisted hopping. The stable states are characterized by an asymmetric double well pseudopotential $U(A)$ with angular frequency of the metastable state $\omega_{A_{1,3}}$ and positive-valued angular frequency of the unstable state at the barrier $\omega_{A_{2}}$ defined through the relation $\omega_{A_{1,3}}^{2}=$ $M_{0}^{-1} U^{\prime \prime}\left(A_{1,3}\right)$ and $\omega_{A_{2}}^{2}=M_{0}^{-1}\left|U^{\prime \prime}\left(A_{2}\right)\right|$. Thus, considering the particle of mass $M_{0}$ moving in a double well quasipotential, the rate coefficients $K^{+}$and $K^{-}$are the forward and backward rates, respectively:

$$
K^{ \pm}=\nu_{ \pm} \exp \left(\frac{-\Delta U_{ \pm}}{D}\right)
$$

where $\Delta U_{ \pm}$denote the threshold energies for activation and $\nu_{ \pm}$are the corresponding pre-factors.

The inverse of the Kramers escape rate is the escape time from one well to another. The two times might be very different [17, 43]. To measure the different properties, we compute the average persistence or residence time $T_{1,3}$ on the attractor with limit cycle amplitude $A_{1,3}$ as:

$$
\begin{aligned}
& T_{1}=T\left(A_{1} \rightarrow A_{3}\right)=\frac{1 / K^{+}}{1 / K^{+}+1 / K^{-}}, \\
& T_{3}=T\left(A_{3} \rightarrow A_{1}\right)=\frac{1 / K^{-}}{1 / K^{+}+1 / K^{-}} .
\end{aligned}
$$

We wish to emphasize here the special character of both the coordinate $A$ and of the quasipotential $U$, see Fig. 3. In fact $A$ is the amplitude of the oscillations approximated by Eq. (11), and therefore it represents the amplitude of an approximated orbit, rather that a single point. Also, the quasipotential $U$ is a (a)

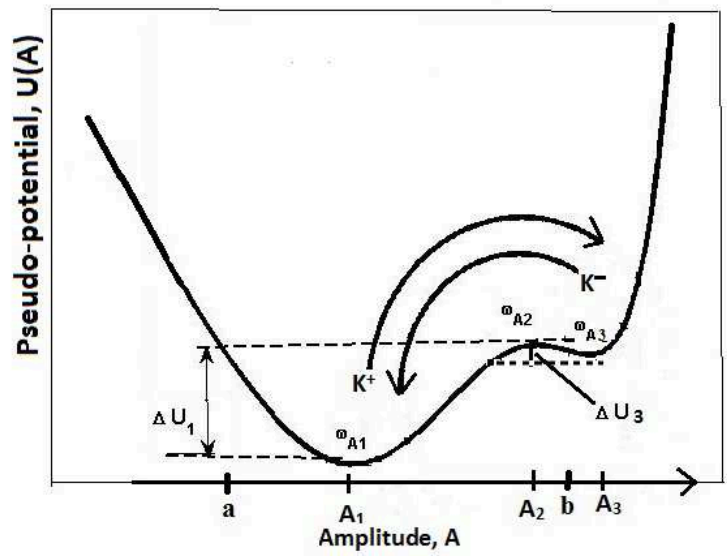

(b)

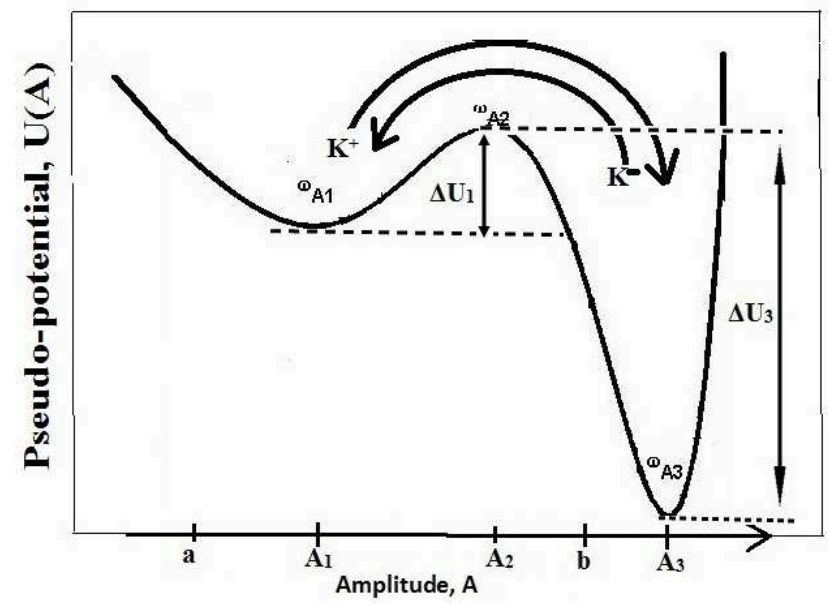

FIG. 3. Schematic illustration of the escape process in an asymmetric metastable potential. Escapes occur via the forward rate $K^{+}$and the backward rate $K^{-}$, respectively, while $\Delta U_{1,3}$ are the corresponding activation energies.

Lyapunov function that characterizes the asymptotic behavior of the nonequilibrium state [19], not a bona fide potential. Therefore Fig. 3 is, at this stage, a pictorial description of the process. However, we will show in the next subsection that a function playing the role of an activation energy (i.e., determining the rate of rare escapes in the low noise intensity limit), can be analytically retrieved.

\section{B. Stationary probability distribution}

In the quasi-harmonic regime, on the assumption that the noise intensity is small, it is convenient to use a change of variables suggested by the approximation Eq. (11), i.e. $x=$ $A \cos \theta$ and $\theta=\varphi+t$ (we assume $\Omega=1$ ). The instantaneous amplitude $A(t)$ and phase $\varphi(t)$ are given by the following effective Langevin equation [17, 18, 21, 33]: 

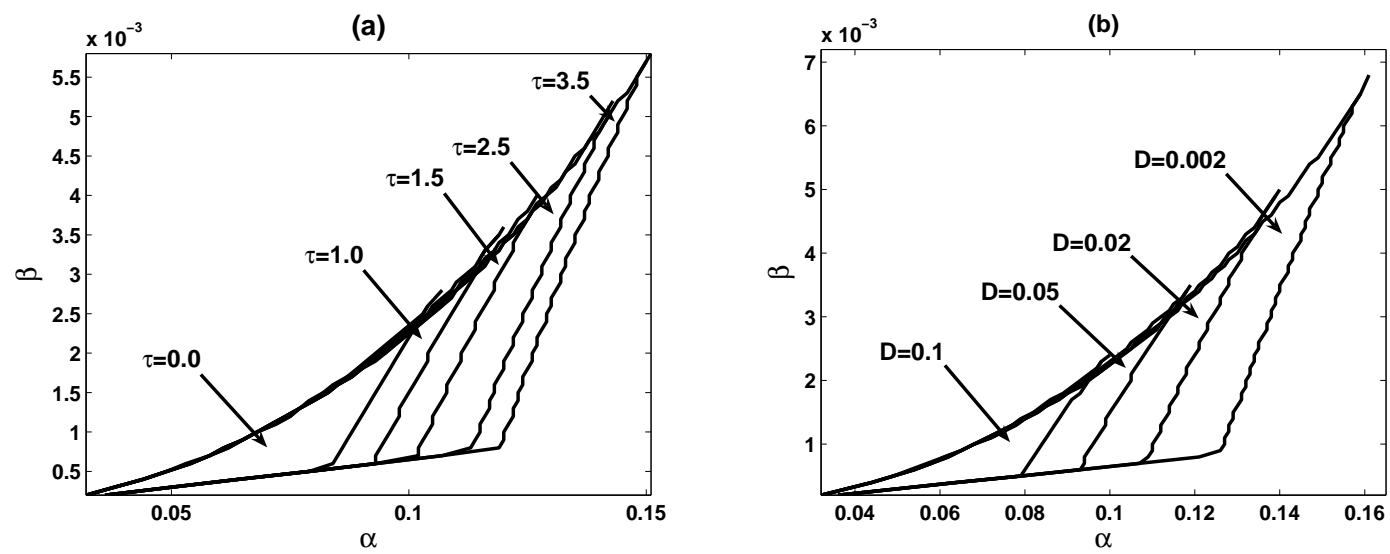

FIG. 4. Effect of the noise and of the correlation time $\tau$ on the boundary between the region of one and three limit-cycle solutions in the parametric $(\alpha, \beta)$-plane for the stationary probability current governed by Eq. 13) as per the analytic solution Eq. (18). (a) The effect of correlation time $\tau$ for $D=0.1$; (b) The effect of noise intensity $D$ for correlation time $\tau=0.1$.

$$
\begin{aligned}
& \frac{d A}{d t}=\frac{\mu A}{128}\left[64-16 A^{2}+8 \alpha A^{4}-5 \beta A^{6}\right]+\frac{D}{2 A\left(1+\tau^{2}\right)}+\xi_{A}(t)=-\frac{d U}{d A}+\xi_{A}(t) \\
& \frac{d \varphi}{d t}=\xi_{\varphi}(t),
\end{aligned}
$$

here $\xi_{A}(t)$ and $\xi_{\varphi}(t)$ represent the noise sources, and $U$ is an effective potential or quasipotential:

$$
U=-\frac{\mu A}{128}\left[32 A^{2}-4 A^{4}+\frac{4}{3} \alpha A^{6}-\frac{5}{8} \beta A^{8}\right]-\frac{D}{2\left(1+\tau^{2}\right)} \ln A .
$$

Contrary to the case of uncorrelated sources, a rigorous derivation of the noise characteristics cannot be obtained, for the additional variable $\eta$ introduces an extra dimension. However, $d A$ does not depend on $\varphi$, thus we assume that we can develop a probability density for $A$, rather than a joint density for A and $\varphi$. For $D \neq 0$, we also assume that Eq. (14b) amounts to an equation for the coordinate $A$ driven by corre- lated noise and we ignore the fact that it represents an orbit rather than a single point.

Consequently, we treat the effect of noise as in the case of a standard double well potential associated to a driven oscillator [44]. In conclusion, the probability density function $p\left(A, t \mid A_{0}, t_{0}\right)$ of the averaged amplitude equation is assumed to be governed by the condition that the probability flux $S_{A}$ vanishes, i.e.:

$$
p(A): S_{A}=0 \rightarrow D_{1}^{A} p(A)-\frac{\partial}{\partial A}\left(D_{2}^{A, A} p(A)\right)=0
$$

where

$$
\begin{aligned}
D_{1}^{A} & =\frac{\mu A}{128}\left[64-16 A^{2}+8 \alpha A^{4}-5 \beta A^{6}\right]+\frac{D}{2 A\left(1+\tau^{2}\right)} \\
D_{2}^{A, A} & =\frac{D}{2\left(1+\tau^{2}\right)}
\end{aligned}
$$

Hence, the stationary solution is:

$$
p(A)=N A \exp \left[\mu \frac{1+\tau^{2}}{512 D}\left(256 A^{2}-32 A^{4}+\frac{32}{3} \alpha A^{6}-5 \beta A^{8}\right)\right],
$$

where 

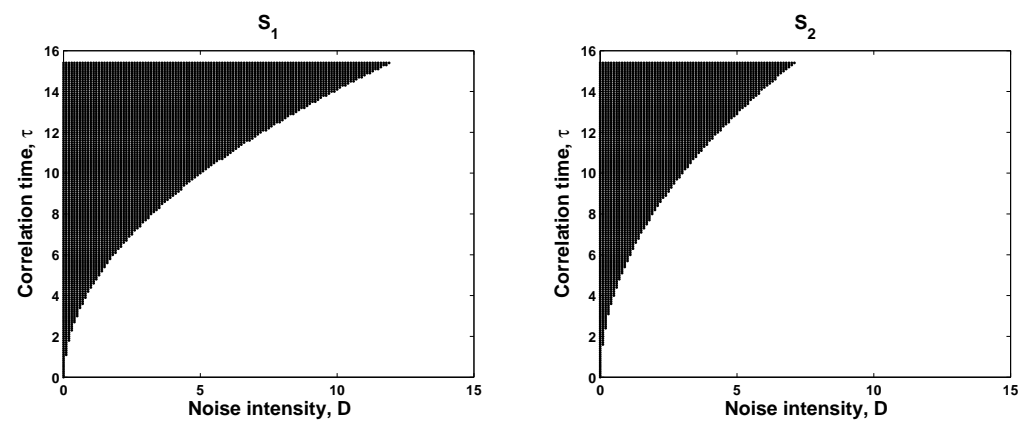

$\mathrm{S}_{3}$
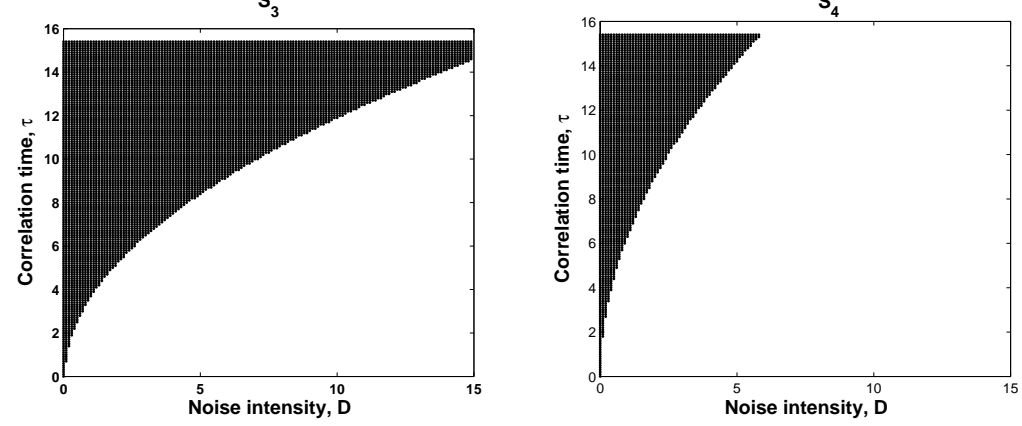

$\mathrm{S}_{5}$
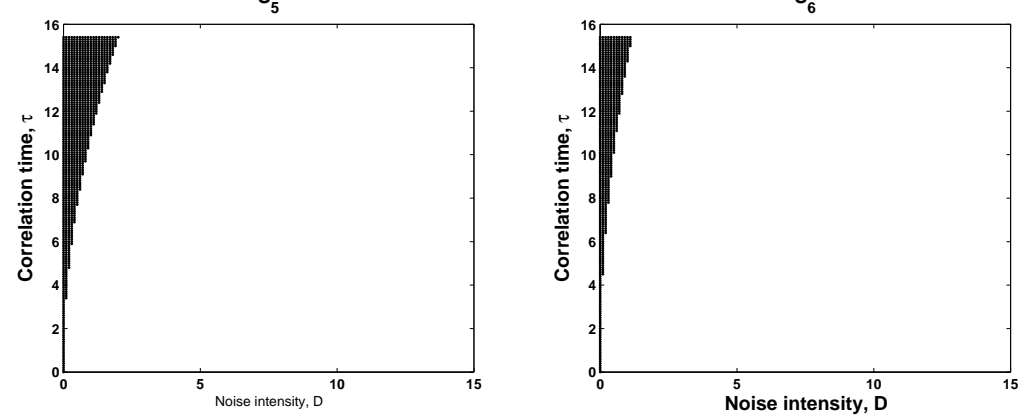

FIG. 5. Stochastic P-Bifurcation in the parameter plane $(D, \tau)$ in the case where the two stable frequencies are equal, $\Omega_{1}=\Omega_{3}$. The dotted area represents the parameter region where two sable orbits coexist. Parameters of the system are $\mu=0.01$ and $S_{i}(i=1,2,3,4,5,6)$

$$
N=\left\{\int_{0}^{+\infty} A \exp \left[\mu \frac{1+\tau^{2}}{512 D}\left(256 A^{2}-32 A^{4}+\frac{32}{3} \alpha A^{6}-5 \beta A^{8}\right)\right]\right\}^{-1}
$$

is the normalization constant.

Equations (18]19) show that in the limit $\tau \rightarrow 0$ the colored noise $\eta(t)$ tends to a white noise [27]. It is important to note that the transitions between the unimodal and the bimodal stationary probability densities are also referred to as the noise induced transitions, and stochastic bifurcation discussed here is closely connected to the noise induced transition [18]. The probability distribution is in general very asymmetric, therefore for most the parameters $\alpha$ or $\beta$ one can localize the probability function around a single orbit. The peaks of the probability distribution can be located deriving the logarithm of the probability distribution, $\partial[\ln p(A)] / \partial A=0$. Thus, to determine the extrema of the distribution Eq. (18) amounts to determine the roots of the equation:

$$
1-\frac{A_{k}^{2}}{4}+\alpha \frac{A_{k}^{4}}{8}-\frac{5 \beta A_{k}^{6}}{64}+\frac{D}{\mu A_{k}^{2}\left(1+\tau^{2}\right)}=0
$$

For $D=0$, the amplitude equation (20) coincides with the deterministic amplitude equation [17]. Generally this equation can admit three positives roots [17, 18] varying the parameters $\alpha, \beta, \mu$, and $D$ the number of the real roots of Eq.(20) change. The effects of the parameters in the system Eq. (18[19) can be seen as a type of a stochastic P-bifurcation [22], i.e a sudden change of the probability density function.

In Fig. 4, we report the influence of the correlation time $\tau$ and the noise intensity $D$ on the birhythmic behavior; in particular we show in Fig. 4 a) the effect of the correlation time 

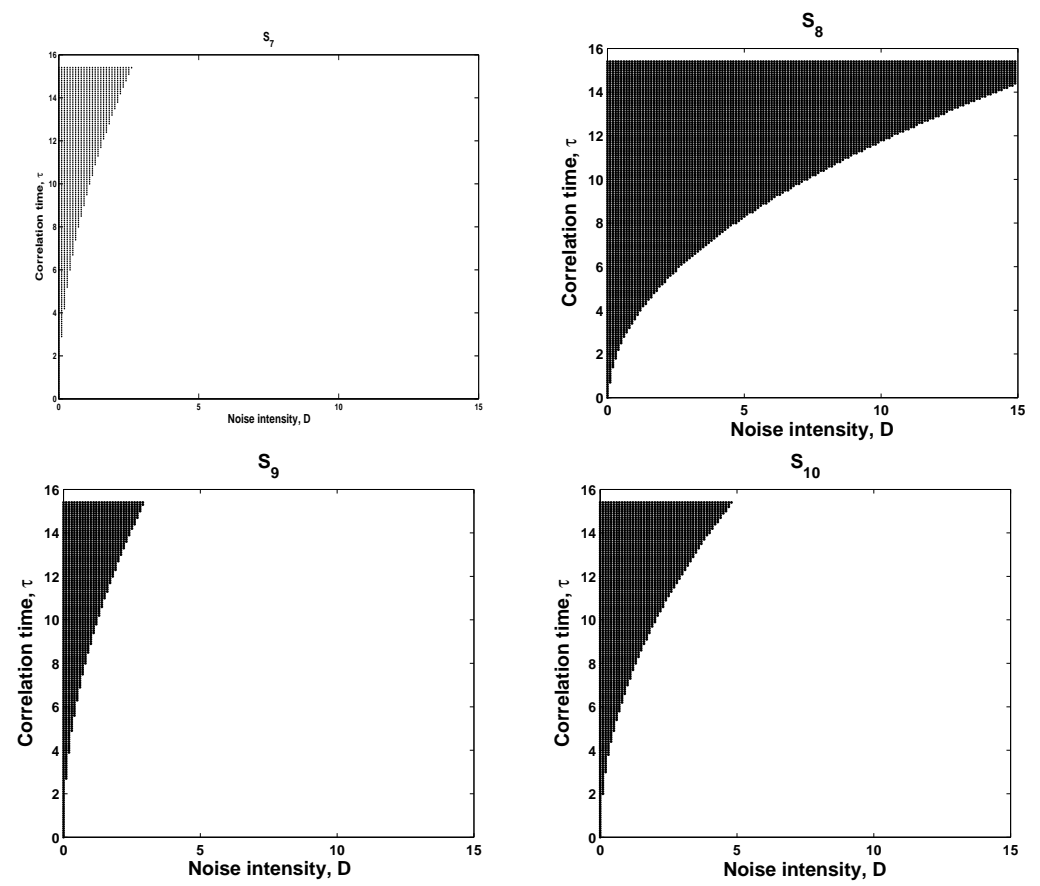

FIG. 6. Stochastic P-Bifurcation in the parameter plane $(D, \tau)$ in the case where the two stable frequencies are different, $\Omega_{1} \neq \Omega_{3}$. The dotted area represents the parameter region where two sable orbits coexist. Parameters of the system are $\mu=0.01$ and $S_{i}(i=7,8,9,10)$

for fixed $D=0.1$, and in Fig. 4(b) the effect of noise intensity for fixed correlation time $(\tau=0.1)$ as predicted by the analysis of Eq.(20). From the Figure, it is clear the opposite effect of the noise intensity $D$ and of the correlation time $\tau$, as the region of birhythmicity increases when $\tau$ increases, and decreases when $D$ increases.

In the same vein to determine the effects of such noise, we represent in Figs. $5[6$ the bifurcation diagram for the system parameter plane $(D, \tau)$ respectively in the case where the two stable frequencies are equal $\left(\Omega_{1}=\Omega_{3}\right)$ and different $\left(\Omega_{1} \neq \Omega_{3}\right)$. The domain of the existence of three limit cycles decreases when the correlated time $\tau$ and the noise intensity $D$ increase, and disappears when these parameters become large enough. One finds the same phenomena for all the set of parameters $S_{i}$.

\section{COMPARISON WITH NUMERICAL SIMULATIONS}

To check the validity of the approximations behind the analytic treatment that has led to the probability density function (18), we have performed numerical simulations of the Langevin dynamics (3) with the numerical scheme to be described in Subsect. IVA.

\section{A. Numerical algorithm for colored noise}

There are several methods and algorithms to solve stochastic differential equations [38], as the implicit midpoint rule with Heun and Leapfrog methods or faster numerical algorithms such as the stochastic version of the Runge-Kutta methods and a quasisymplectic algorithm [38-40]. The starting point is the Box-Muller algorithm [41] to generate exponentially correlated colored noise distributed random variable $\eta_{\Delta t}$ from the Gaussian white noise and two random numbers $a$ and $b$ which are uniformly distributed on the unit interval $[0,1]$.

To simulate the exponentially correlated colored noise, Eqs.(3) are replaced with the following equations [42]:

$$
\begin{aligned}
& \dot{x}=u, \\
& \dot{u}=\mu\left(1-x^{2}+\alpha x^{4}-\beta x^{6}\right) u-x+\eta, \\
& \dot{\eta}=-\lambda \eta+\lambda g_{w},
\end{aligned}
$$

where $g_{w}$ is a Gaussian white noise:

$$
\begin{aligned}
& \left\langle g_{w}(t)\right\rangle=0 \\
& \left\langle g_{w}(t) g_{w}\left(t^{\prime}\right)\right\rangle=2 D \delta\left(t-t^{\prime}\right) .
\end{aligned}
$$

Combining Eq. (21c) and Eqs. (22) one gets an exponentially correlated colored noise $\eta$. To numerically solve Eqs.(21), we use the Box-Mueller algorithm [41] to generate a Gaussian white noise $g_{w}$ from two random numbers $a$ and $b$, which are uniformly distributed on the unit interval, as in Refs. [17, 18].

The equations have been integrated halving the step size until consistent results are obtained (the problem is particularly delicate in the proximity of an absorbing barrier [40]). Furthermore, the procedure has been calibrated with a standard activation barrier to retrieve the Kramers escape rate [12], as modified by the correlation time $\tau$ [27]. In the end, we have find that a step size $\Delta t=0.0001$ is generally appropriated, 

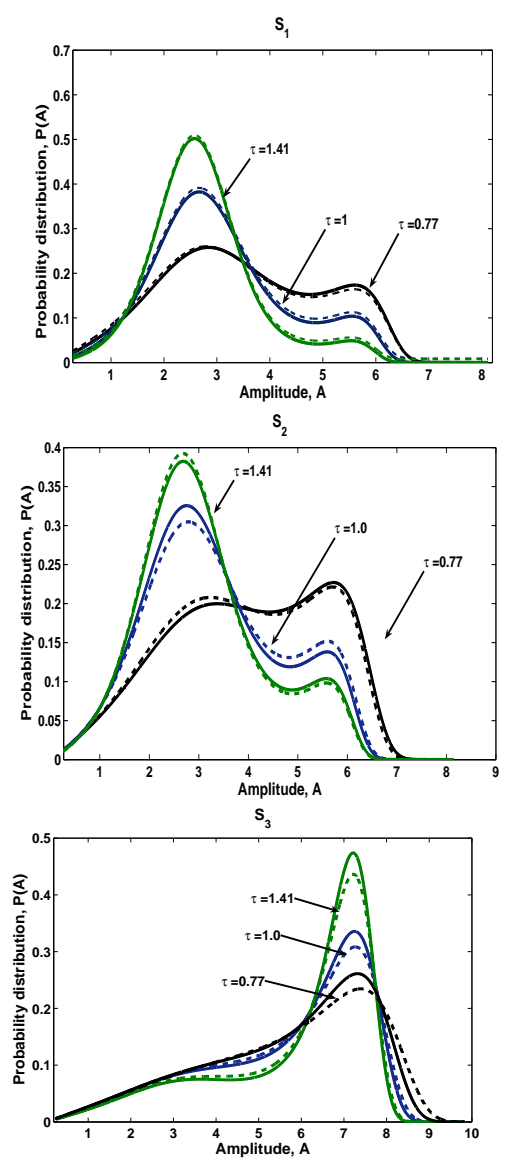

$\mathrm{S}_{4}$

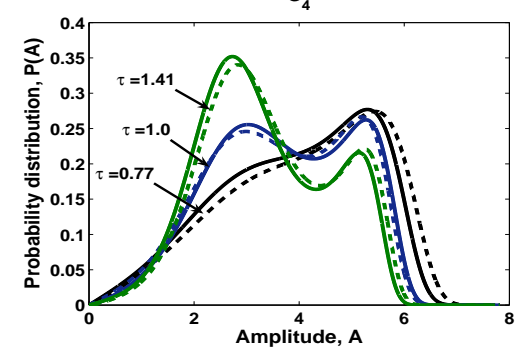

$S_{5}$

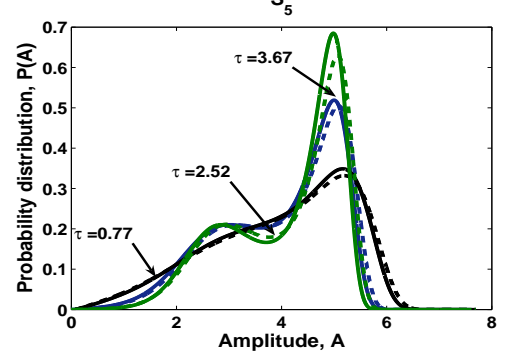

$S_{6}$

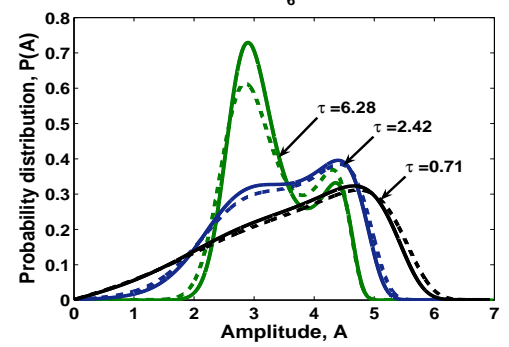

FIG. 7. Probability distributions of the amplitude A for different values of the correlation times $\tau$ when the frequencies of both attractors are identical, i.e $\Omega_{1} \cong \Omega_{3} \cong 1$. Solid lines denote the analytical results, while dashed lines denote numerical results. Parameters of the system refer to $S_{i}$ of Table $\square$ with $\mu=0.01, D=0.01$
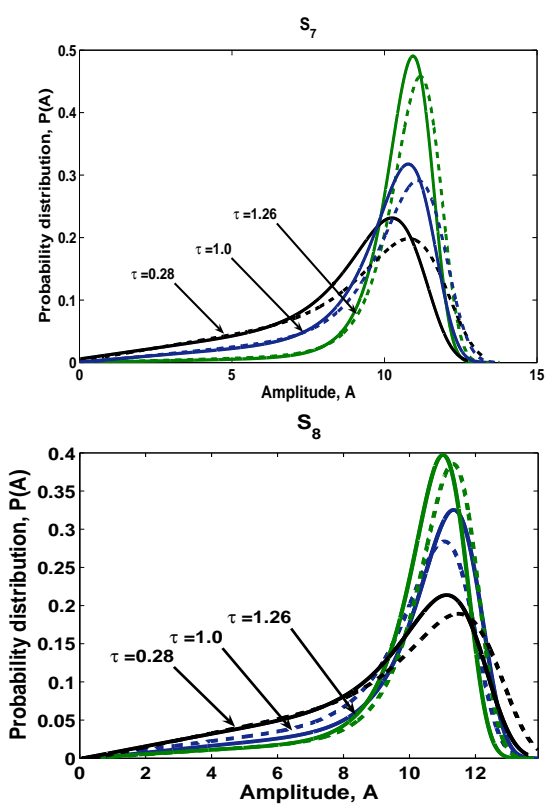

$s_{9}$

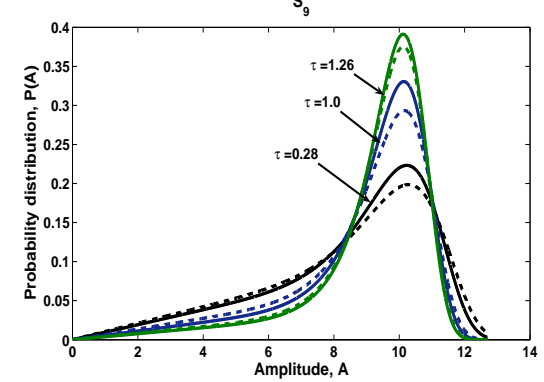

$S_{10}$

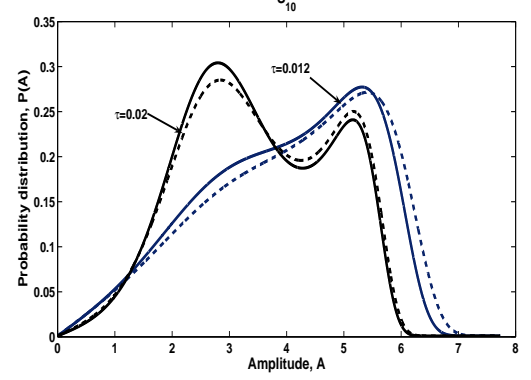

FIG. 8. Probability distributions of the amplitude A for different values of the correlation times $\tau$ when the frequencies of the attractors are different, $\Omega_{1} \neq \Omega_{3}$. Solid lines denote the analytical results, while dashed lines denote numerical results. Parameters of the system refer to $S_{i}$ of Table II with $\mu=0.01, D=0.4$

but in few cases it has been necessary an even smaller step. Moreover, we have averaged the results over 200 realizations, that ensures converge within few percents. This scheme has been employed to check the validity of the approximations behind the analytic treatment that has led to the probability density function (18).

In Fig. 7, we plot the behavior of the probability distribution $p$ as a function of the amplitude $A$ for low noise intensity $D=0.01$ and several values of the correlation time $\tau$, when the frequencies of both attractors are similar, i.e. 


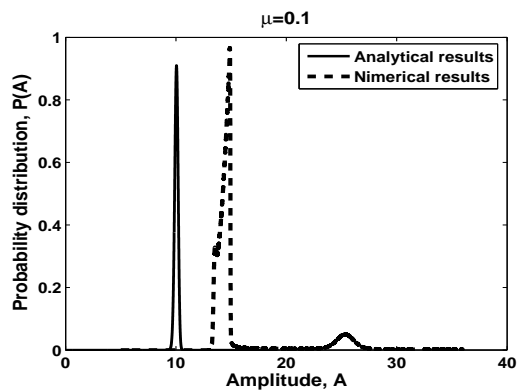

$\mu=0.01$
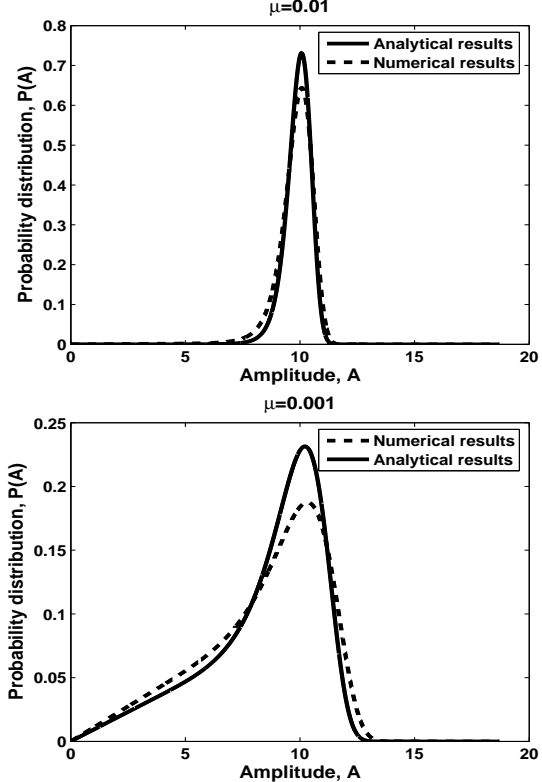

$\mu=0.0001$

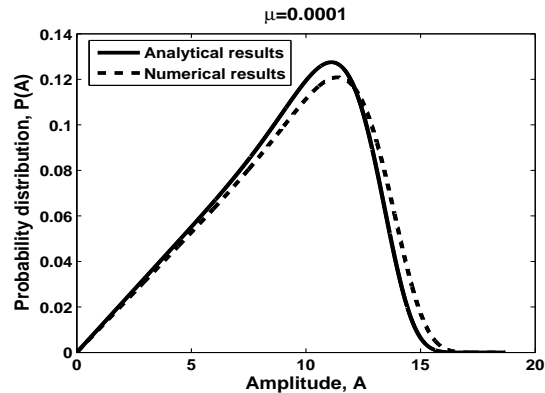

FIG. 9. Probability distributions versus the amplitude A for different values of the coefficient $\mu$ when the frequencies of both attractors are different, $\Omega_{1} \neq \Omega_{3}$. Solid lines denote the analytical results, while dashed lines denote numerical results. Parameters of the system refer to corresponding to $S_{8}$ of Table II with $D=0.05$ and $\tau=0.0001$

(i) We begin with a correlation time that is substantially zero $(\tau=0.001)$ and $T_{1}$ is larger than $T_{3}$; in such conditions the attractor of the limit-cycle amplitude $A_{1}$ is more stable than the limit-cycle amplitude $A_{3}$. (This correspond to the set of parameters $S_{1}, S_{3}, S_{5}$.) Increasing the correlation time one observes the symmetric case $T_{1} \simeq T_{3}$ : both attractors are equivalent and the system is in a symmetric bistable double well, i.e. it
$\Omega_{1} \simeq \Omega_{3} \simeq 1$. It appears that the system is more likely found at two distinct distances from the origin, the essential feature of birhythmicity. In fact, for the parameters here considered, there are two attractors in the deterministic system, see Table [. However, as underlined in the previous Section, we expect that noise can destroy birhythmicity (see Fig. 47. In fact, for short correlation times, the amplitude has sometimes a single peak (parameters $S_{4,5}, 6$ ). We also notice that the correlation noise tends to restore the birhythmic behavior, and in fact for the largest values of the noise correlation time $\tau$ we always find a two-peak solution. The probability distribution $p$ is asymmetric for the set of parameters $S_{1,2,3,4,5}$. Although the correlation time varies from the values $\tau=0.77$ to $\tau=1.47$, this asymmetric property of the density probability distribution $p$ persists. In all these configurations, the comparison between the analytic and numerical results is acceptable.

Fig. 8 shows the variation of the probability distribution density $p$ versus the amplitude $A$ when the frequencies of both attractors are different, (i.e. $\Omega_{1} \neq \Omega_{3}$, see Table 【i) for $D=$ 0.4 and different values of the correlation times $\tau$. Also in this case it is evident that the correlation time $\tau$ tends to stabilize a second orbit, and thus gives rise to a birhythmic behavior.

Finally, in Fig. 9 we show the probability distribution function when the frequencies that characterize the attractors are different varying the nonlinear parameter $\mu$. The Figure demonstrates that the analytic and numerical estimates appear to be quite close, except at high levels of the parameter $\mu$.

To measure the effect of the correlation time on the stability of the two solutions, let us return to Eq. (13) that shows the connection between the average persistence (or residence) times $T_{1,3}$ on the attractor with limit cycle amplitude $A_{1,3}$ and the corresponding escape rates determined by the quasipotential (12). The analytical and numerical behaviors of the residence times versus $\tau$ are reported in Figs. 10 and 11, for $\Omega_{1} \simeq \Omega_{3}$ and $\Omega_{1} \neq \Omega_{3}$, respectively. In Fig. 10, three relevant cases can be found for the first occurrence $\left(\Omega_{1} \simeq \Omega_{3}\right.$, Table 【:

has approximately the same probability to stay in one or the other basins. As the correlation time is further increased, one finds the reverse situation: $T_{1}<T_{3}$, and the first attractor becomes less stable. Thus, for larger correlation times the system is more likely to stay on the limit-cycle attractor $A_{3}$. It is interesting to notice that the agreement between the approximated analysis and the numerical simulations is satisfying for all val- 
$\mathrm{s}_{1}$

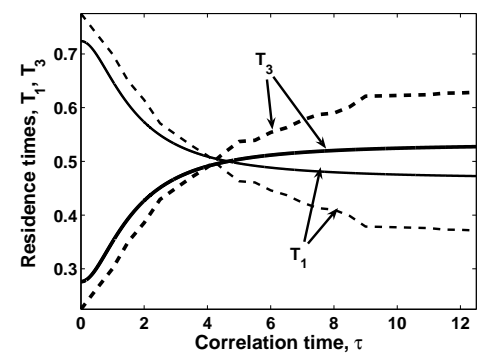

$\mathrm{S}_{3}$

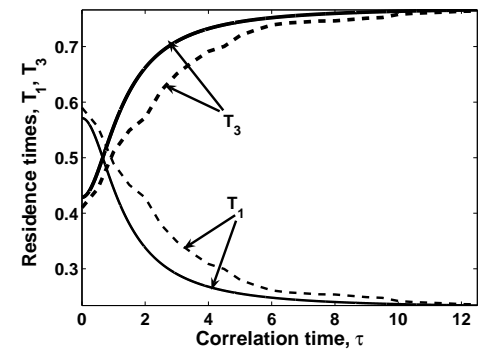

$\mathrm{S}_{5}$

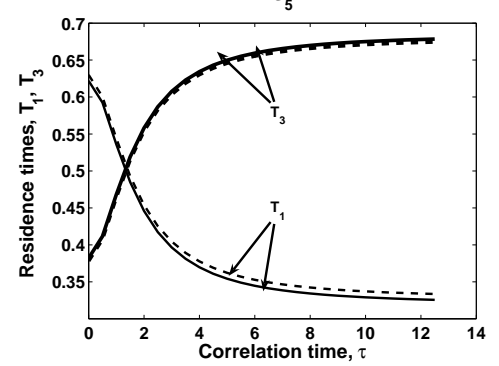

$\mathrm{S}_{2}$

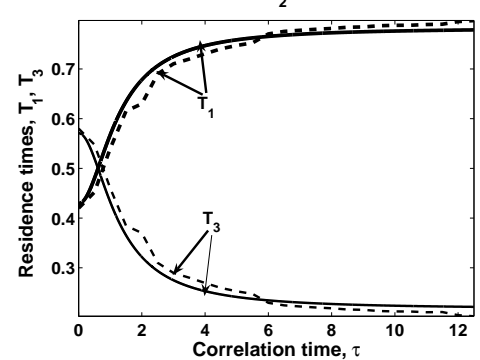

$\mathrm{S}_{4}$

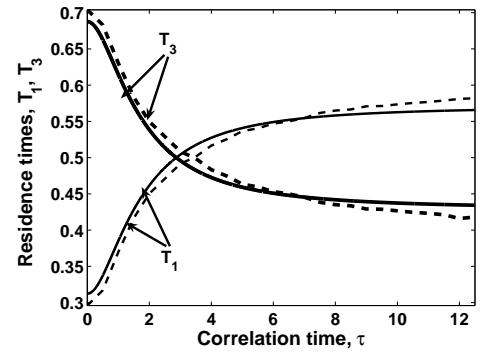

$\mathrm{S}_{6}$

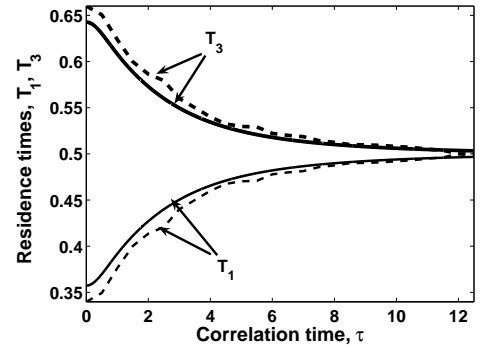

FIG. 10. Residence times versus the correlation time $\tau$ for different values of the parameter $S_{i}=(\alpha ; \beta)$ when the frequency are almost identical $\left(\Omega_{1} \cong \Omega_{3} \cong 1\right.$ ). Solid lines denote the analytical results, while dashed lines denote numerical results. Parameters of the system refer to $S_{i}$ of Table $\square$ with $\mu=0.01, D=0.01$.

ues of the correlation time, also when the correlation is comparable to the period of the cycle, $2 \pi / \Omega \simeq 2 \pi$.

(ii) In the second case when the correlation time is increased from zero $(\tau>0.001)$ the attractor orbit $A_{3}$ is first more stable than the attractor orbit $A_{1}$ (this correspond to the set of parameters $S_{2}, S_{4}$ ), and becomes less stable with the increases of the correlation time $\tau$. In other words, this is the reverse transition respect to the first case (i);

(iii) In the third case (corresponding to the case $S_{6}$ ), the attractor orbit $A_{3}$ is much more stable $\left(T_{3}>T_{1}\right)$, as in (ii). However, as the correlation time increases, one observes the transition between asymmetric and symmetric cases, $T_{1} \simeq T_{3}$ that seems to be the asymptotic solution for extremely large correlation times.

In Figure 11, we instead investigate the case when the two attractors are characterized by different frequencies (Table $\amalg$, we find still another case (for the attractors $S_{7}, S_{8}$, and $S_{9}$ ): the inner attractor corresponding to the orbit $A_{1}$ is more stable when the correlation time is small, and becomes progressively even more stable when the correlation time is increased.
In conclusion, the effect of the correlation time on the relative stability of the two attractors seems to be very differentiated across the parameter space. However, despite the very different behaviors, the approximated analysis seems to capture the main physical effects.

To outline the behavior as a function of the oscillator parameters, we show in Figs. 12 the behavior of the activation energies for increasing values of the noise correlation time ( $\tau=0.001,1, \pi, 2 \pi)$ when the parameter $\alpha$ increases form 0.095 to 0.135 for $\beta=0.002$. In this range of parameters, the solution exhibits (almost) identical frequencies of the two attractors $\left(\Omega_{1,3} \simeq 1\right)$. In this change of the parameter $\alpha$ the role of the two activation energies is exchanged: at low $\alpha$ the lowest energy is the inner energy, while at the other limit the outer activation energy is the lowest. However, the approximation (15) is valid all through the parameter change for correlation times that are as long as the period of the oscillations $(\tau \simeq 2 \pi / \Omega)$.

Figs. 13 describes the same type of analysis keeping a constant value of the parameter $\alpha=0.12$ and varying the parameter $\beta$. Also in this case the effective trapping energy undergoes a drastic change and the roles of the two barrier are 

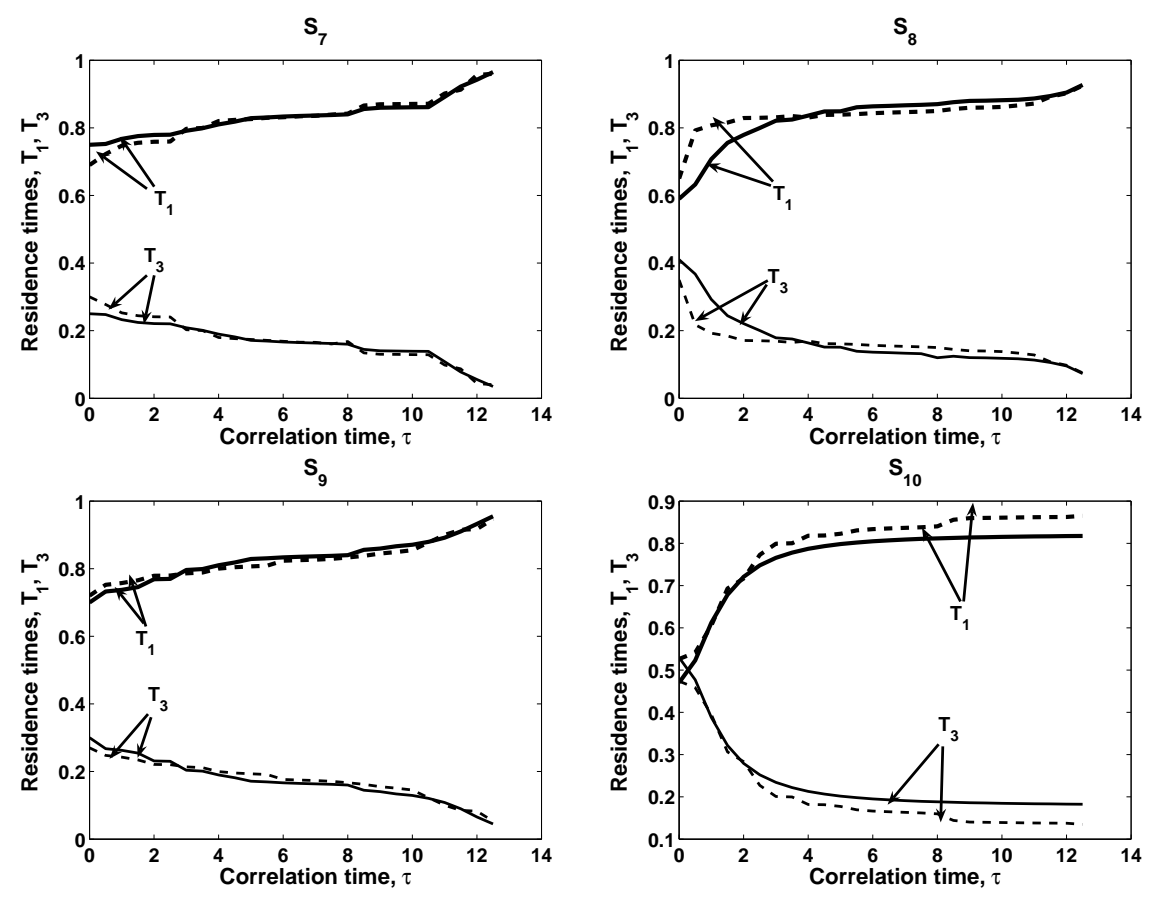

FIG. 11. Residence times versus the correlation time $\tau$ for different values of the parameter $S_{i}=(\alpha ; \beta)$ when the frequency are different ( $\Omega_{1} \neq \Omega_{3}$ ). Solid lines denote the analytical results, while dashed lines denote numerical results. Parameters of the system refer to $S_{i}$ of Table [1] with $\mu=0.01, D=0.2$.
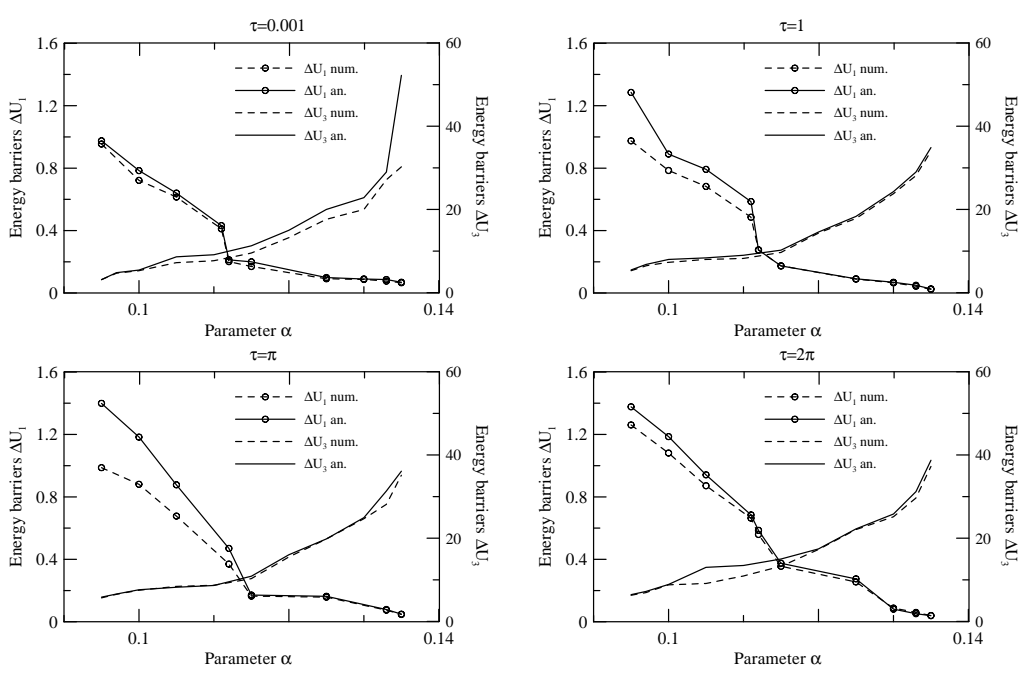

FIG. 12. Energy barriers $\Delta U_{1,3}$ versus $\alpha$. The lines with symbols refer to the energy barrier of the inner orbit ( $\Delta U_{1}$, left axis), while the lines without symbols to the outer orbit activation energy $/ \Delta U_{3}$, right axis). Solid lines denote the estimates as per Eq. (15), while dashed lines denote numerical results. The other parameters of the system are $\mu=0.01$ and $\beta=0.002$.

exchanged. Thus, the numerical results of Figs. 12]13 confirm that the analytic approach leading to the quasipotential 15 is very accurate in describing the system.

\section{CONCLUSIONS}

The main conclusion of this work has been the existence of a drastic change (a P-bifurcation [22]) of the probability distribution function of a birhythmic van der Pol like oscillator subject to exponentially correlated noise. We have particularly investigated the evolution of the modified van der Pol 

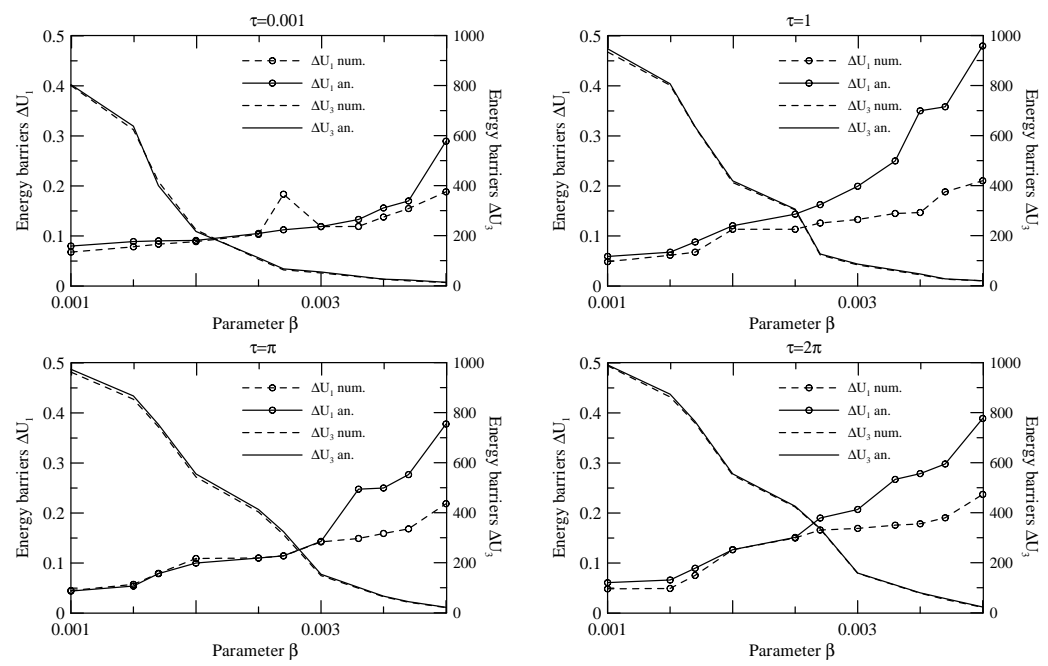

FIG. 13. Energy barriers $\Delta U_{1,3}$ versus $\beta$. The lines with symbols refer to the energy barrier of the inner orbit ( $\Delta_{1}$, left axis), while the lines without symbols to the outer orbit activation energy $/ \Delta U_{3}$, right axis). Solid lines denote the analytical estimates as per Eq. [15, while dashed lines denote numerical results. The other parameters of the system are $\mu=0.01$ and $\alpha=0.12$.

oscillator in the region where birhythmicity occurs. The characteristics of the birhythmic properties are strongly influenced by the nonlinear coefficients as well as the noise intensity and correlation. We have performed a detailed analysis of the bifurcation diagrams in the parameters plane, and it is evident that the noise intensity and the correlation time can be treated as bifurcation parameters. An approximated solution of the Langevin equation based on the Fokker-Planck equation in the quasiharmonic regime [33] gives the stationary probability density $p(A)$ of the instantaneous amplitude. With this approach, a stochastic bifurcation, a qualitative change of the stationary solution, occurs when the intensity or correlation of the noise is changed. In this system stochastic P-bifurcations [22] correspond to the appearance and disappearance of one of the maxima of the distribution of the amplitudes $p(A)$. The boundary of the existence of multi-limit-cycle solutions, in the parametric $(\alpha, \beta)$-plane, is affected in different manners by the noise intensity $D$ and the correlation times $\tau$ : As expected for the standard analysis of correlated noise, to increase the correlation time amounts to decrease the effect of the noise intensity. The simulations have confirmed that the approximated (obtained through stochastic averaging) Fokker-Planck equation well describes this type of birhythmic oscillators. We therefore expect that real systems (either biological or electronic circuits) governed by birhythmic van der pol type equation are very sensitive to changes in the correlation time of the noise. In fact, the appearance of correlated noise can drastically change the structure of the attractors, that could possibly be a signature of the presence of significatively correlated noise. For instance, the noise correlation in Fig 11 or Fig. 12 entails that the most stable attractors might become another one, with a different amplitude (Fig. 11) or both different amplitude and frequency (Fig, 9, parameter set $S_{10}$ ).

Summing-up, we have found that the quasiharmonic balance is (surprisingly) effective in predicting the features of the full system, as checked with numerical simulations, even when the correlation time is comparable to the period of the solutions, Fig. 8, (and this is even more surprising) when the two solutions are characterized by different frequencies. However, we have found (not surprisingly) that when the parameter $\mu$, that tunes the nonlinearity, is increased, the predictions are less accurate, see Fig. 9 It is therefore tempting to draw the general conclusion that the quasi-harmonic balance is an effective tool for self-oscillatory systems, and even for birhythmic systems characterized by two different frequencies, if the nonlinear terms are kept at bay. Of course, no matter however relevant it might be considered the van der Pol oscillator, one should also bear in mind that the conclusion is based on a specific set of simulations on a single system. As such, the conclusion should be taken with due care.

\section{ACKNOWLEDGMENTS}

The authors thank E. Tafo Wembe for enriching contributions. 
[1] Blondel A. Amplitude du courant oscillant produit par les audions générateurs. Comptes Rendus Hebdomadaires des Seances de l'Academie des Sciences 1919;169: 943 - 948.

[2] Van der Pol B. A theory of the amplitude of free and forced triode vibrations. Radio Rev. (London) 1920; 1:701710, 754762.

[3] Ginoux J-M, Letellier C. Van der Pol and the history of relaxation oscillations: Toward the emergence of a concept. Chaos 2012; 22:0231201-15.

[4] Jewett ME, Forger DB, Kronauer RE. Revised Limit Cycle Oscillator Model of Human Circadian Pacemaker. J. Biol. Rhythms 1999; 14: 493-499.

[5] Anishchenko VS, Astakhov A, Neiman A, Vadivasova T, Schimansky-Geier L. Nonlinear Dynamics of Chaotic and Stochastic Systems: Tutorial and Modern Developments. Berlin: Springer; 2007.

[6] Phillips JK, Chen PY, Robinson PA. Probing the mechanisms of chronotype using quantitative modeling., J. Theor. Biol. 2010; 25: 217-227.

[7] Curie P. Équations réduites pour le calcul des mouvements amortis. La Lumiere Electrique 1891; 31: 201-209, 270-276, 307-362.

[8] Hayashi C. Nonlinear Oscillations in Physical Systems. New York: Mc-Graw-Hill; 1964.

[9] Noise in nonlinear dynamical system, Moss F, McClintock PVE (editors). Cambridge: Cambridge University Press, 1989.

[10] Zakharova A, Vadivasova T, Anishchenko V, Koseska A, Kurths J. Stochastic bifurcations and coherencelike resonance in a selfsustained bistable noisy oscillator; Phys. Rev. E 2010: 81; 0111061-6.

[11] Berglund N, Gentz B. On the Noise-Induced Passage through an Unstable Periodic Orbit II: General Case. SIAM Journal on Mathematical Analysis 2014; 46(1): 310-352.

[12] Kramers HA.Brownian motion in a field of force and the diffusion model of chemical reactions. Physica (Amsterdam) 1940; 7: 284-304.

[13] Van Kampen NG. Escape and splitting probabilities in diffusive and non-diffusive Markov processes. Prog. Theor. Phys. 1978; 64: 389-401.

[14] Yamapi R, Filatrella G, Aziz-Alaoui MA. Global stability analysis of birhythmicity in a self-sustained oscillator. Chaos 2010; 20: 0131141-12.

[15] Yamapi R, Filatrella G, Aziz-Alaoui MA, Cerdeira HA. Effective Fokker-Planck equation for birhythmic modified van der Pol oscillator. Chaos 2012; 22,:0431141-9.

[16] Ghosh P, Sen S, Riaz SS, Ray DS. Controlling birhythmicity in a self-sustained oscillator by time-delayed feedback. Phys. Rev. E 83 2011; 0362051-9.

[17] Cheage Chamgoue A, Yamapi R, Woafo P. Dynamics of a biological system with time-delayed noise. Eur. Phys. J. Plus 2012; 127: 59.

[18] Cheage Chamgoue A, Yamapi R, .Woafo P. Bifurcations in a Birhythmic biological system with time-delayed noise. Nonlinear Dynamics 2013; 73: 2157-2173.

[19] Graham R, Tél T. Weak-noise limit of Fokker-Planck models and nondifferentiable potentials for dissipative dynamical systems. Phys. Rev. A 31 1985; 1109-1122.

[20] Kautz RL. Thermally induced escape: the principle of minimum available noise energy. Phys. Rev. A 1988; 38:2066-2080; J. Appl. Phys. 1994; 76: 5538-5544.
[21] Mbakob Yonkeu R, Yamapi R, Filatrella G, Tchawoua C. Pseudopotential of birhythmic van der Pol type systems with correlated noise. Uunpublished 2014.

[22] Arnold L. Random Dynamical System. Berlin: Springer, 2003.

[23] McNamara B, Wiesenfeld K. Theory of stochastic resonance. Phys. Rev. 1989; A 39: 4854-4869.

[24] Gammaitoni L, Hanggi P, Jung P, Marchesoni F. Stochastic resonance. Rev. Mod. Phys. 1998; 70: 223-287.

[25] Addesso P, Filatrella G, Pierro V. Characterization of escape times of Josephson junctions for signal detection. Phys. Rev. E 2012; 85: 0167081-15.

[26] Risken H, Debnath G, Moss F, . Leiber Th, Marchesoni F. Holes in the two-dimensional probability density of bistable systems driven by strongly colored noise. Phys. Rev. A1990; 42: 703710 .

[27] Hanggi P, Jung P. Adv. Chem. Phys. 1995; 89: 239-326.

[28] Fiasconaro A, Spagnolo B. Stability measures in metastable states with Gaussian colored noise. Phys. Rev. E 2009; 80: 0411101-6.

[29] Spagnolo, B., Valenti D., Fiasconaro, A.: Noise in ecosystems: A short review. Math. Biosci. Eng. 2004; 1: 185.

[30] Kaiser F. Coherent modes in biological systems. In: Illinger $\mathrm{KH}$, editor. Biological efects of nonionizing radiationr, A.C. Symp. Series; 1981, p.157.

[31] Roberts J, Spanos P. Stochastic averaging: An approximate method of solving random vibration problems. Int. J. NonLinear Mech. 1996; 21: 111-134.

[32] Bag BC, Hu C-K. Escape through an unstable limit cycle: Resonant activation (correlated noise). Phys. Rev. E 2006; 73: 0611071-4

[33] Xu Y, Gu R, Zhang H, Xu W, DuanJ, Stochastic bifurcations in a bistable DuffingVan der Pol oscillator with colored noise. Phys. Rev. E 2011; 83: 0562151-7.

[34] Valenti D, Guarcello C, Spagnolo B, Switching times in longoverlap Josephson junctions subject to thermal fluctuations and non-Gaussian noise sources. Phys. Rev. B 2014; 89: 214510115.

[35] Enjieu Kadji HG, Chabi Orou JB, Yamapi R, Woafo P. Nonlinear dynamics and strange attractor in the biological system. Chaos, Solitons and Fractals 2007; 32: 862-882.

[36] Enjieu Kadji HG, Yamapi R, Chabi Orou JB. Synchronization of two coupled self-excited systems with multi-limit cycles. Chaos 2007; 17:0331131-14.

[37] Borland L. Ito-Langevin equations within generalized thermostatics. , Phys. Lett. A 1998; 245: 67-72.

[38] Mannella R. Integration of stochastic differential equation on computer. Int. J. Mod. Phys. C 2002; 13: 1177-1194.

[39] Mannella R. Absorbing boundaries and optimal stopping in a stochastic differential equation. Phys. Lett. A 1999. 254: 257262.

[40] Mannella R. Quasisymplectic integrators for stochastic differential equations. Phys. Rev. E 2004; 69: 0411071-8.

[41] Knuth DE. The art of Computer Programming, Vol. 2. Reading MA: Addison-Wesley; 1969.

[42] Fox RF, Gatland IR, Roy R, Vemuri G. Fast, accurate algorithm for simulation of exponentially correlated colored noise. Phys. Rev. A 1998; 38: 5938-5940.

[43] Stambaugh C, Chan HB. Noise-activated switching in a driven nonlinear micromechanical oscillator. Phys. Rev. Lett. 2006; 97: 1106021-4.

[44] Jung P. Periodically driven stochastic systems. Phys. Rep. 1993; 234: 175 - 295. 